\title{
The proto-black hole concept in GRB 101023 and its possible extension to GRB 110709B.
}

\author{
A.V. Penacchioni, G.B. Pisani \\ Erasmus Mundus Joint Doctorate IRAP PhD. Student. Università La Sapienza, Piazzale Aldo \\ Moro, 5, 00185 Rome, Italy. \\ E-mail: ana.penacchioni@icra.it, giovanni.b.pisani@gmail.com \\ R. Ruffini, C.L. Bianco, L. Izzo, M. Muccino \\ Dip. di Fisica, Sapienza Università di Roma and ICRA, Piazzale Aldo Moro 5, I-00185 Roma, \\ Italy. \\ E-mail: [ruffini, bianco, luca.izzo, marco.muccino]dicra.it
}

From the spectral analysis and the light curve of GRB 101023, a clear evidence of two spectral components has been outlined [1]. The first episode has been fit by a black body plus powerlaw spectral model. The temperature changes with time following a broken power-law. The second episode appears to be a canonical GRB. Using the Amati [2] and Atteia [3] relations we determined the cosmological redshift to be $z=0.9 \pm 0.08$ (stat.) \pm 0.2 (sys.). This source appears to be a twin of GRB 090618 [4]: the first episode has been defined as the proto-black hole and the second one presents the characteristics of a canonical GRB. We are exploring the possibility to extend these considerations to GRB 110709B. GRB 110709B has been detected by Konus Wind and Swift [5] in gamma and X rays, and by Gemini and GROND telescopes in the optical bands. It is a very particular GRB, since Swift has triggered twice [7]. Its light curve presents a two-episode structure, one that begins $40 \mathrm{~s}$ before the trigtime and lasts $\sim 100 \mathrm{~s}$, and another that starts $485 \mathrm{~s}$ after the trigtime and lasts $\sim 380 \mathrm{~s}$. This separation between the two episodes is the largest seen up to date.

Gamma-Ray Bursts 2012 Conference -GRB2012,

May 07-11, 2012

Munich, Germany 

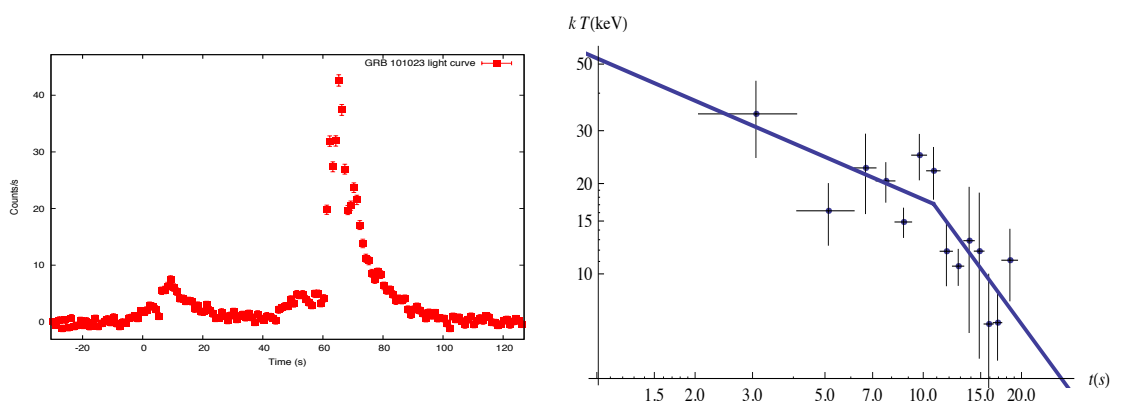

Figure 1: (a) GRB 101023 count light curve from Fermi GBM detector (bin time of $1 \mathrm{~s}$ ). The time is given with respect to the GBM trigtime of 22:50:04.73 UT, 23 Oct, 2010. (b) Fit of the observed temperature kT of the BB component with a broken power-law model. The indices of the first and second power-law are $\alpha=-0.47 \pm 0.34$ and $\beta=-1.48 \pm 1.13$, respectively. The break occurs at $11 \mathrm{~s}$ after the trigtime.

\section{GRB 101023}

The $23^{\text {rd }}$ October, 2010 the Fermi GBM [8] detector triggered a source quite similar to GRB 090618. The burst was also detected by BAT [5] on board Swift satellite at UT 22:50:12. The Swift XRT detector $[9,10]$ has triggered on this source $88 \mathrm{~s}$ after the BAT trigger. GRB 101023 was also detected by the Wind instrument on board Konus satellite, in the energy range (10 - 770) $\mathrm{keV}$ [11]. The inferred location is in complete agreement with that determined by Swift and Fermi. Moreover, there have been detections in the optical band by the Gemini telescope [12].

We used the data from the second $\mathrm{NaI}$ detector (see Fig 1a) in the range $8-440 \mathrm{KeV}$. Then we proceeded with its time resolved spectral analysis with the programs XSPEC and RMFIT. The GBM light curve shows two major pulses. The first one starts at the Fermi GBM trigtime and lasts $25 \mathrm{~s}$. The topology of this curve leads to think that this may not be the case of a canonical GRB, in analogy with GRB 090618 [4]. The second pulse starts 45s after the trigtime and lasts 44s. This second emission does have all the characteristics which describe a canonical GRB. We analysed this source within the fireshell model [13].

The redshift of this source is unknown, due to the lack of data in the optical band. However, to constrain it, we employed different methods. First we made use of the Amati relation [2], under the hypothesis that only the second episode is a long GRB. We computed the values of Ep,i and Eiso for different given values of $\mathrm{z}$. We found that the Amati relation is fulfilled by episode 2 for $0.3<z<1.0$. Then we used an empirical method, following Atteia [3] and Pelangeon [14], which consists in determining a pseudo-redshift from the GRB spectral properties (for deatils about the procedure see [3]). We applied this treatment to Episode 2, introducing the spectral parameters from the Band model in the Cosmos website ${ }^{1}$ and obtained a value for the redshift of $z=0.9 \pm 0.08$ (stat.) \pm 0.2 (sys.). The systematic error between the pseudo- $z$ determined with this procedure and the actual redshift $z$ for the GRBs in which it has been measured is of the order of $30-50 \%$ (see [3], [14], [19], [1]).

To identify the nature of Episode 1, we fit the data with a black body (BB) plus a power-law component (see Table 1). Although the spectral index of the power-law is very hard, it remains

\footnotetext{
${ }^{1}$ http://cosmos.ast.obs-mip.fr/projet/v2/fast computation.html
} 
Table 1: Fit of Episode 1 with a Black Body plus a power-law model.

\begin{tabular}{cc}
\hline Parameter & Value \\
\hline $\mathrm{kT}(\mathrm{keV})$ & $12 \pm 2$ \\
$\gamma$ & $-1.9 \pm 0.2$ \\
Amp (po) & $(1.97 \pm 1.59) \times 10^{-3}$ \\
Amp (bb) & $(1.3 \pm 0.3) \times 10^{-4}$ \\
$\chi^{2}$ & 0.82 \\
\hline
\end{tabular}
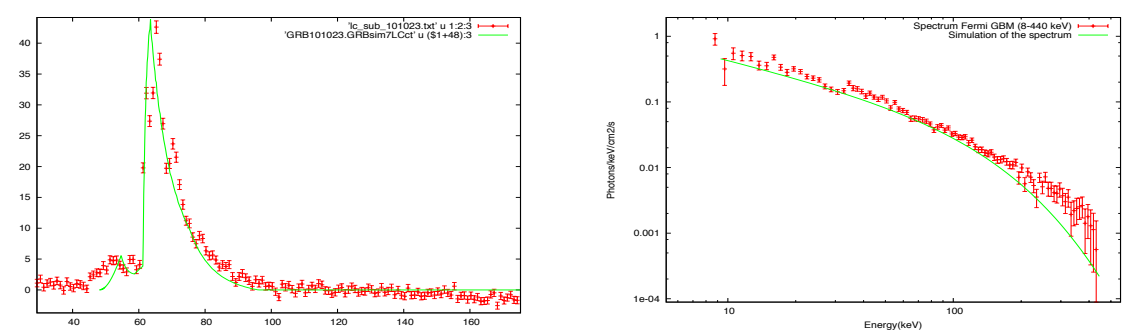

Figure 2: Simulation of the light curve and spectrum of GRB 101023 with GRBsim.

below the upper limits (see fig. 4 of [1]). We do not discard the possibility that there is a cutoff at higher energies, but as we do not have any LAT observation, we cannot specify at which energies this occur. We plotted the temperature of the BB component as a function of time, for the first $20 \mathrm{~s}$ of emission (see Fig. 1b). We note a strong evolution which, according to Ryde [15] can be reproduced by a broken power-law behavior. In order to simulate the light curve we made use of a numerical code called GRBsim [16]. This numerical code simulates a GRB emission by solving the fireshell equations of motion, taking into account the effect of the EQuiTemporal Surfaces (EQTS). We made the simulation for Episode 2. We found, at the transparency point, a value of the laboratory radius of $1.34 \times 10^{14} \mathrm{~cm}$, a theoretically predicted temperature that after cosmological correction gives $k T_{t h}=13.26 \mathrm{keV}$, a Lorentz Gamma factor of $\Gamma=260$, a P-GRB laboratory energy of $2.51 \times 10^{51} \mathrm{erg}$ and a P-GRB observed temperature of $28 \mathrm{keV}$. We adopted a value for the dyadosphere energy of $E_{d y a}=1.8 \times 10^{53} \mathrm{erg}$ and a baryon load of $B=3.8 \times 10^{-3}$. The errors of these values are $\sim 10 \%$. The simulated light curve and spectrum of Episode 2 are shown in Figure 2.

\section{GRB 110709B}

GRB 110709B has been detected by Suzaku [17] and Swift [18] satellites, and by on-ground telescopes like GROND and Gemini. The light curve shows two emissions, separated by a time interval of ten minutes (see Fig 3 a). This is the first source in which BAT has triggered twice. Unfortunately, as is the case also for GRB 101023, we do not know its redshift, so we inferred it from the empirical methods mentioned above. We obtained a range for $\mathrm{z}$ of $0.6<z<1.35$, so we took the mean value, $z=0.975$. 

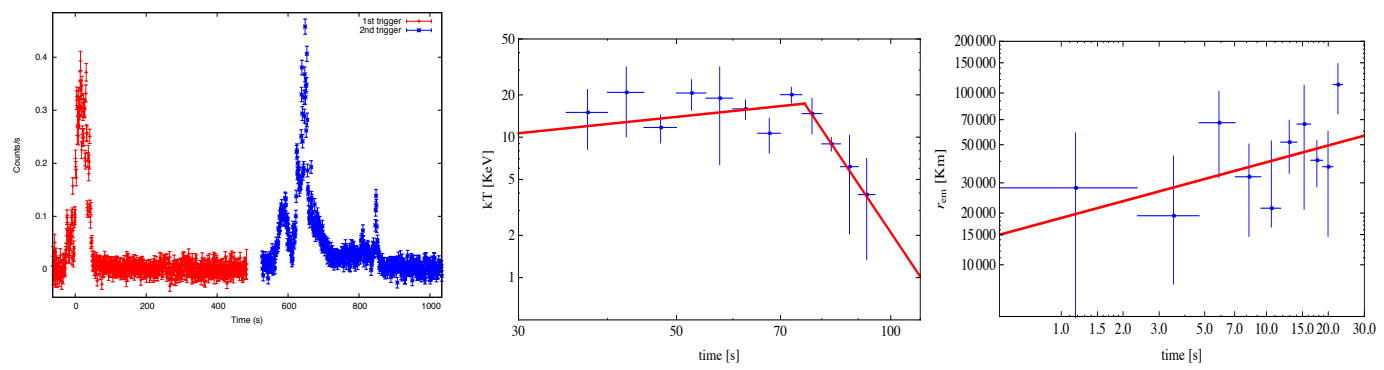

Figure 3: (a) GRB 110709B light curve obtained from the Swift BAT detector in the band $(15-150) \mathrm{keV}$. The red (blue) points correspond to the first (second) trigger. (b) Evolution of the kT of the BB plus powerlaw model of Episode 1. The solid line is the broken power-law fit. (c) Evolution of the radius of the progenitor. It increases with time as a power-law $a x^{b}$, with $a=(1.8 \pm 1.3) \times 10^{4}$ and $b=0.3 \pm 0.2$.
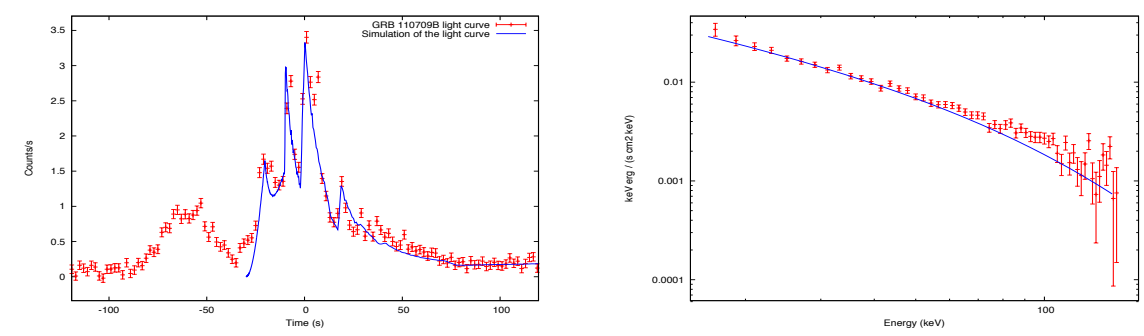

Figure 4: Simulation of the light curve and spectrum of Episode 2 with GRBsim.

The first episode (corresponding to the first trigger) is well fit by a black body plus a powerlaw component. The temperature of the black body decreases with time following a broken powerlaw (see Fig 3 b). We determined the value of the progenitor radius, which increases with time following a power-law (see Fig. 3c). These figures present preliminary results. Details will be reported in Penacchioni et al. (in preparation).

The second emission seems to be a canonical GRB. We simulated the light curve and the spectrum with the numerical code GRBsim (see Fig. 4) and obtained the following values for the parameters of the model: Lab Radius $=1.012 \times 10^{14} \mathrm{~cm}, \Gamma=358, E_{P G R B}=1.8 \times 10^{-2} E_{d y a}$, PGRB obs. temperature $=35 \mathrm{keV} /(1+z)$. There is though a slight discrepancy at high energies between the data points and the simulation. To estimate the uncertainties of these parameters, we perform the simulation many times changing slightly the parameters to explore the range in which the simulation is still compatible with the data. It turns out that such uncertainties are $\sim 10 \%$.

\section{Conclusions}

We conclude that, in the context of the fireshell model, GRB 101023 and GRB 090618 share similar properties. They both present what we defined as the 'proto-black hole' emission. It is also appropriate to remark that this new kind of sources does not present any $\mathrm{GeV}$ emission. We are proceeding to the identification of additional sources belonging to this family, as it can happen with 
GRB 110709B, which we are still analyzing. We have already found the thermal component in the first seconds of emission, which again follows a broken power-law behavior.

\section{References}

[1] A.V. Penacchioni, R. Ruffini, L. Izzo, et al., Evidence for a proto-black hole and a double astrophysical component in GRB 101023, A\&A 2012 (538) A58

[2] L. Amati., The Ep,i-Eiso correlation in gamma-ray bursts: updated observational status, re-analysis and main implications, MNRAS 2006 (372) 233

[3] J-L. Atteia, A simple empirical redshift indicator for gamma-ray bursts, A\&A 2003 (407) L1

[4] L. Izzo, R. Ruffini, A.V. Penacchioni, et al., A double component in GRB 090618: a proto-black hole and a genuinely long $G R B, A \& A$, 2011, (arXiv: 1202.4374)

[5] C. J. Saxton, S.D. Barthelmy, W.H. Baumgartner, et al., GRB 101023A: Swift detection of a burst GCN 113632010

[6] M. Nardini, F. Olivares and J. Greiner, GRB 101023A: GROND detection of the optical-NIR afterglow, GCN 113692011

[7] S.D. Barthelmy, D.N. Burrows, J.R. Cummings, et al., GRB 110709B: Swift detection of renewed activity at T+11 minutes., GCN 12124, 2011

[8] M. S. Briggs, GRB 101023A: Fermi GBM observation, GCN 11376, 2010

[9] K. L. Page, C.J. Saxton, GRB 101023A: Swift-XRT Team Refined Analysis, GCN 11368, 2010

[10] D. Burrows, J. Hill, J. Nousek, et al., The Swift X-Ray Telescope Space Science Reviews, 2005, (120) 165

[11] S. Golenetskii, R. Aptekar, D. Frederiks, et al., Konus-Wind observation of GRB 101023A GCN 11384,2010

[12] A.J. Levan, D. Perley, P. DÕAvanzo, GRB 101023A: Gemini discovery of optical afterglow, GCN 11366,2010

[13] R. Ruffini, Proceedings of the Twelfth Marcel Grossmann Meeting onGeneral Relativity, World Scientific, Singapore, 2011

[14] A. Pelangeon, J.-L. Atteia, D.Q. Lamb, et al., An improved redshift indicator for Gamma-Ray Bursts, based on the prompt emission, Gamma-ray bursts in the Swift Era, ed. S. S. Holt, N. Gehrels, \& J. A. Nousek (New York), AIP, 2006

[15] F. Ryde, The Cooling Behavior of Thermal Pulses in Gamma-Ray Bursts, ApJ, 2004, (614) 827

[16] C. L. Bianco \& R. Ruffini, Exact versus Approximate Solutions in Gamma-Ray Burst Afterglows, ApJ, 2005a, (633) L13

[17] N. Ohmori, M. Akiyama, M. Yamauchi, et al., GRB 110709B : Suzaku WAM observation of the prompt emission, GCN 12172, 2011

[18] J. R. Cummings, S. D. Barthelmy, D. N. Burrows et al., GRB 110709B: Swift detection of a burst, GCN 12122, 2011

[19] A. Pelangeon, J.-L. Atteia, Y. E. Nakagawa,, et al., Intrinsic properties of a complete sample of HETE-2 gamma-ray bursts. A measure of the GRB rate in the Local Universe, A \& A, 2008, (491) Issue 1, 157-171 